\title{
CONSIDERACIONES SOBRE LA CRISIS DE LOS AÑOS 68-69
}

\author{
MANUEL ABILIO RABANAL ALONSO \\ Universidad de Alicante
}

\begin{abstract}
El año que va desde la muerte de Nerón al comienzo del imperio de Vespasiano representa una encrucijada militar y socioeconómica. Sus protagonistas merecen el calificativo de triunfadores coyunturales. Cuando llegar al poder depende de la mejor oferta económica o de la donación de puestos administrativos, nada puede haber políticamente consistente. Esta es una de las razones fundamentales de la crisis del año conocido como «de los cuatro emperadores».
\end{abstract}

L'an qui va depuis la mort de Néron jusqu'au commencement de l'empire de Vespasien représente un carrefour militaire et socioéconomique. Ses protagonistes méritent le qualificatif de triomphateurs conjoncturaux. Quand l'arrivée au pouvoir dépend de la meilleure offre économique ou de la concession de places administratives, rien ne' peut' être consistent en politique. Celle-ci est une des raisons fondamentales de la crise de l'an connu comme «des quatre empereurs».

Es teoría comúnmente expresada o compartida por la gran mayoría de los historiadores que esta crisis demuestra que un emperador podía llegar al poder sin ser romano ni italiano, situación insólita hasta este momento que tratamos. Y, más aún, que Roma ya no era la clave política en exclusiva del Imperio, sino una ciudad más, aunque, eso sí, con el privilegio de ser la ciudad-madre. Tratamos en el presente trabajo de llegar a matizar algunos aspectos que quizá no sean los fundamentales, pero sí interesantes y que nos proporcionan muchas luces para entender mejor este período de crisis.

Los problemas básicos en torno a esta crisis se centran en tres aspectos fundamentales:

a) Causas de planteamiento de la crisis.

b) Demostraciones de los acontecimientos y paralelos.

c) Soluciones a la crisis.

\section{a) Causas del planteamiento de la crisis}

El primer problema que se nos presenta es la falta de sucesores en la línea dinástica de Nerón. Por otro lado uno de los males de mayor importancia y ya tradicional en la historia del Imperio es el hecho de que el ejército se manifiesta como el poder fundamental y desde esta perspectiva puede interpretarse como un auténtico vicio del mismo sistema imperial. De ahí que comiencen a surgir emperadores fuera de Italia y con el apoyo primero y casi exclusivo del ejército. Además la dinastía de los Julio-Claudios y la regionalización de los ejércitos demuestran la importancia creciente de las provincias. 
La clave del comienzo de la crisis está en las provincias occidentales del Imperio.

Si el final del imperio de Nerón se había caracterizado por la persecución directa y constante de la nobleza, ahora la misma nobleza ve en Galba al representante de sus intereses mediante cuya situación van a tratar de salvarse a sí mismos y simultáneamente al Imperio. Esta puede ser una de las razones por las que Suetonio (1) trata de justificar por todos los medios el origen noble del propio emperador Galba, y Tácito (Historias, I, XV) da por la misma razón una gran importancia a la «Nobilitas».

Las luchas a lo largo de este período de crisis se puede concretar en rivalidades de ejércitos, característica del quehacer militar de forma absolutamente preponderante.

La economía del Imperio, como consecuencia de los gastos extraordinarios en ingentes cantidades, motivados por los dispendios del emperador Nerón, hicieron pensar que la solución estaba en una persona que debería ser esencialmente contraria a la condición de su antecesor en el Imperio. La solución, por lo tanto, concretada en la persona de Galba, tendrá dos razones básicas: equilibrio económico y reducción de gastos. Pero estas dos soluciones, que pueden considerarse virtudes económicas, degeneran en avaricia y tacañería. Por ello este planteamiento no va a solucionar la gran crisis económica, sino que generará el descontento de prácticamente todos los ciudadanos y más aún de los soldados, excluyéndose solamente algunos de sus amigos particulares y sus propios libertos. Donde fundamentalmente va a notarse este descontento, como se indica más arriba, es entre los militares; consigue Galba algo tan negativo como la animadversión de los soldados, a quienes niega el pago de lo prometido cuando trataba de acceder al Imperio, según atestigua un texto de SUETONIO (Galba, 16).

Otra de las razones mediante la cual trata de controlar y ejercer un auténtico poder absoluto es la crueldad puesta en práctica contra todos, puesto que en realidad todos los estamentos están descontentos con su actuación política.

Una vez más es Suetonio (Galba, 22) quien hace desde su perspectiva de biógrafo la valoración de si realmente la actuación de Galba puede calificarse como una mezcla de vicios económicos, sociales y políticos o costumbres arraigadas desde su propio origen de patricio y, en definitiva, representante de la alta aristocracia romana, que ostenta la totalidad del poder; o, dicho de otro modo, si sus planteamientos son simples frutos personales o resultado de la pertenencia a una clase determinada.

El primer paso en la carrera política de Galba hacia el Imperio está evidentemente apoyado por otra serie de provincias al lado de Hispania, que se constituye como el punto de partida. Esto queda de manifiesto en una moneda (2), en

(1) Galba, 2-3: Expone el árbol genealógico de Galba, para demostrar que llega incluso a entroncar con Júpiter y Parsifae. Además hace un recuento de sus allegados más próximos, todos ellos pertenecientes a la más alta aristocracia romana.

(2) Se trata de un denario, fechado en el año 68, procedente de Hispania, aunque sin localización exacta. En el anverso aparece un busto de la Libertad con la siguiente inscripción: LIBERTAS RESTITU$\mathrm{TA}$; en el reverso figura inscrito S(enatus) $\mathrm{P}$ (opulus Q(ue) R(omanus). Esta moneda se incluye en el libro de H. MATTINGLY, 1926, 292, n. ${ }^{\circ} 12$, y en el de M. Mc CRUM y A. G. WOODHEAD, 1961, 36, n. ${ }^{\circ} 25$. 
la que a Galba se le califica de «restaurador de la libertad» y además como el «promotor de la alianza entre Hispania y las Gallias» en otra moneda también procedente de Hispania (3). En esta segunda moneda se habla también de una auténtica «victoria», que sería el signo final para la pacificación del Imperio. En otra moneda (4), ésta procedente de la Gallia, se considera a Galba como el «salvador de todo el género humano» y en otra (5), de Roma, se califica al emperador de «cuidador oficial de todos los ciudadanos». En una inscripción (6) de Roma se repite la titulación de Galba como «restaurador de la libertad».

Hay que pensar que, aparte de su propia propaganda política, quizá toda esta serie de calificativos tratan de justificar el deseo unánime por parte de todos, militares y civiles, de volver a encontrar el auténtico sentido de equilibrio en el Imperio, con el fin de eliminar los problemas de la gran crisis que se está padeciendo.

El paso siguiente en la historia imperial tendrá las mismas motivaciones que el anterior nombramiento de Galba. Si este emperador se negó en un momento determinado a pagar a los soldados lo prometido, ahora se buscará la solución en un nuevo emperador, que ha montado su propaganda política haciendo hincapié en su generosidad; así, una vez más, se hablará de una auténtica paz universal (7). Y también concretando un ámbito de consenso militar, razón esencial del nombramiento del emperador Otón (8).

Puede observarse que el término «libertas» es utilizado tanto por quien ostenta el poder como por quienes se oponen a la situación de mando del candidato-emperador. La «libertas» significaría constitucionalmente la vuelta a

(3) Es un denario. En el anverso aparecen bustos representando a Hispania y a la Gallia con la siguiente inscripción: CONCORDIA HISPANIARUM ET GALLIARUM. En el reverso tiene una representación de la Victoria e inscrito: VICTORIA P(opuli) R(omani). Está publicada por H. MATTINGLY, 1926, p. 293-T, y Mc CRUM y A. G. WOODHEAD, 1961, 36, n. ${ }^{\circ} 26$. La fecha corresponde al año 68 (imperio de Galba).

(4) Se trata de un denario. En el anverso tenemos la siguiente inscripción: SALUS GENERIS HUMANI. En el reverso aparecen inscritas las letras SPQR., que corresponden a «Senatus Populusque Romanus». Esta moneda ha sido recogida por H. MATTINGLY, 1926, 297, n. ${ }^{\circ} 31$, y también publicada por M. Mc CRUM y A. G. WOODHEAD, $1961,36, n{ }^{\circ} 27$. La fecha correspondiente es el año 68 , imperio de Galba.

(5) Es un sestercio. En el anverso aparece la cabeza de Galba laureada y con la inscripción SER. GALBA IMP. CAESAR AUG. TRIB. P. En el reverso leemos EX S. C. OB CIVES SER VATOS. Está publicada por H. MATTINGLY, 1926, 318, n. ${ }^{\circ} 63$, y por M. Mc CRUM y A. G. WOODHEAD, 1961, 36, n. ${ }^{\circ} 28$. La fecha es diciembre del año 68 (Galba).

(6) CIL, VI, 471 = ILS, I, 238. El texto de la inscripción es el siguiente: «imaginum domus Aug. cultorib. signum Libertatis restitutae Ser. Galbae imperatoris Aug. curatores anni secundi: C. Turranius Polybius L. Calpurnius Zena C. Murdius Lalus C. Turranius Florus C. Murdius Demosthenes s. p. d. d. dedic. idib. Octobr. C. Bellico Natale P. Cornelio Scipione Asiatico cos».

(7) Es un «aureus», procedente de Roma. Puede leerse en el anverso lo siguiente: IMP. M. OTHO CAESAR AUG. TR. P.; esta inscripción circunda la cabeza de Otón. En el reverso aparece la Paz con una rama en la mano derecha y caduceo en la izquierda y se lee PAX ORBIS TERRARUM. La fecha corresponde a los primeros meses del año 69, es decir al imperio de Otón. Fue publicada por H. MATTINGLY, 1926, $364, n .{ }^{\circ} 1$, y recogida también por M. Mc CRUM y A. G. WOODHEAD, 1961, 37, n. 32.

(8) El «consenso militar» aludido queda de manifiesto en una moneda de Tarraco (TarragonaHispania), un as, en cuyo anverso puede verse la cabeza de Vitelio laureada con la siguiente inscripción: A. VITELLIUS IMP. GERMAN. En el reverso aparece el dios Marte con yelmo, lanza en la mano derecha y en la izquierda un estandarte militar rematado en águila e inscrito CONSENSUS EXERCITUUM S. C. Fue publicada por H. MATTINGLY, 1926, 388, n. ${ }^{\circ} 99$, y por M. Mc CRUM y A. G. WOODHEAD, 1961, 37, n. ${ }^{\circ} 36$. Cronológicamente hay que fijarla en el año 69 , meses del mandato de Vitelio. 
la situación creada por Augusto. El Senado quizá lo que en realidad busca es la vuelta al viejo régimen republicano.

Si una de las razones económicas de la crisis estaba en los abusos tributarios impuestos por Nerón a las provincias, Galba tratará de congraciarse con la población eliminando algunos impuestos y poniendo trabas a la actuación de los procuradores imperiales.

Por otro lado sigue en pie la referencia importante a favor de la aristocracia (TACITO, Historias, I, LXXXVIII).

Los intereses fundamentales de Otón se centran en el deseo de contemporización con todos los ejércitos, tratando de evitar la presión de los pretorianos y los requerimientos del Senado. Precisamente, ante esta situación, surge un nuevo problema, que puede concretarse en un cambio de persona para regir los destinos del Imperio Romano. La formación del nuevo emperador Vitelio es esencialmente militar y, por esa razón, su triunfo, una vez más, dependerá del apoyo del ejército.

Si tuviéramos que calificar la acción de Vitelio como emperador, tendríamos que decir que tiene el acierto, sin duda esencial, de lograr un buen entendimiento con el ejército y la crueldad para con el resto de los agentes subversivos que se manifiestan en todos los puntos claves del Imperio (SUETONIO, Vitelio, 13).

La confianza militar puesta en Vitelio queda bien de manifiesto en una moneda procedente de Germania Superior (9). Ya al final de su mandato, el emperador Vitelio va a centrar su actividad en conseguir el apoyo, por otro lado dudoso, mediante donaciones y promesas (SUETONIO, Vitelio, 15). A pesar de todo, surgirá una larga serie de sublevaciones concretadas en los ejércitos de Moesia, Pannonia, Judea y Siria, que van a nombrar a Vespasiano. De nuevo queda de manifiesto que los ejércitos más poderosos son los del Rhin, Danubio y «limes» oriental.

\section{b) Demostraciones de los acontecimientos y paralelos}

Existen varios textos, que, al mismo tiempo que demuestran algunos acontecimientos de la crisis, pueden a veces apoyarse o contradecirse. El análisis de los más importantes nos llevará a un conocimiento más exacto de la situación real del llamado año de los cuatro emperadores.

TACITO (Historias, I, XXXIV) dice textualmente: «Nec diutius Galba cunctactus speciosiora suadentibus accesit». Evidentemente este texto de Tácito se contrapone con lo que dice SUETONIO $($ Galba, 19). Además hay un segundo punto en Tácito en el mismo capítulo citado, que no recoge Suetonio y es la deci-

(9) Es un denario. En el anverso aparecen unas manos entrelazadas y la siguiente inscripción: FIDES EXERCITUM. En el reverso también pueden verse unas manos entrelazadas y la inscripción FIDES PRAETORIANORUM. Con exactitud puede fecharse en los meses del imperio de Vitelio del año 69. Fue publicada por H. MATTINGLY, 1926, 306, n. ${ }^{\circ} 65$, y recogida por M. Mc CRUM y A. G. WOODHEAD', $1961,38,{ }^{\circ}{ }^{\circ} 38$.

(10) En relación con la composición del Senado en este momento son básicos dos artículos de $\mathrm{H}$. HAMMOND, 1957, 74-81, y 1956, 61-133. 
sión de enviar por delante a Pisón, texto recogido en los siguientes términos: «Praemissus tamen in castra Piso...»

En lo que respecta a la relación de Tácito con Plutarco podemos observar una casi total identidad, al menos de contenido, entre el cap. LXXXI deL libro I de las Historias de Tácito y el cap. III de la vida de Otón, de Plutarco.

Por otro lado hay una coincidencia importante entre TACITO ( Historias, I, LXXXIV) y TITO LIVIO (XLIV, XXXIX), en cuanto que Otón habla en el texto de Tácito de forma similar a como lo hace Paulo Emilio en el texto de Tito Livio.

TACITO (Historias, I, LXXXIV) sigue diciendo que el Senado es la cabeza del Imperio y el honor de las provincias. Al fin y al cabo esto constituye una auténtica ficción política, que no tiene que ver demasiado con la realidad, basándose en parte en el hecho de que los personajes más distinguidos de las provincias formaban parte del orden senatorial. En el mismo autor (TACITO, Historias, I, LXXXIV) podemos ver además reminiscencias claras de un discurso de Camilo, que aparece en TITO LIVIO (V, LIV). En ambos textos se refiere la importancia del Senado como elemento de unión y simultáneamente garantizador de la estabilidad política.

Quizá otro de los paralelos más representativos, entre los textos de la época que estudiamos, es el existente en el discurso de Otón, recogido por TACITO (Historias, II, XLVII) y el que nos transmite PLUTARCO (Otón, 15).

Las circunstancias en que se desenvuelve la historia de este año de crisis demuestra la inestabilidad constante y cómo, además y de forma real, cada ejército quiere como emperador a su propio jefe militar. De ello tenemos claras demostraciones en las revueltas prácticamente continuadas, muy especialmente en la Gallia y también en Germania. Otro síntoma evidente de lo dicho tiene como protagonista a los pretorianos, quienes no dudan en apoyar a uno u otro candidato al Imperio, solamente basados en las promesas económicas.

Algunos de los paralelos y diferencias que recogemos aquí nos demuestran que no se puede hablar de unanimidad entre los escritores antiguos. Por supuesto queda claro que la forma de escribir la Historia tiene en todo momento el subjetivismo de las interpretaciones.

Con Galba ha quedado perfectamente claro cuáles son las características y aspiraciones de la aristocracia.

Es básica en este orden de cosas la postura de un historiador italiano (E. MANNI, 1946, 122-156), quien trata de basar su criterio político de la crisis, partiendo de una realidad concreta. Otro historiador (G. MANFRE, 1947, 128) dice textualmente que en este período de crisis se da «un espíritu de libertad en las provincias proponiendo sus derechos contra el Senado, puesto que se apoya en las legiones». En definitiva es una aspiración autonómica por conseguir la libertad y no en función de algún pretendido separatismo. Al fin y al cabo la crisis fue un paso en la evolución política del Imperio Romano, es decir del proceso universal hacia la unificación de las provincias, de acuerdo con un estudio referido a este momento histórico (A. GARZETTI, 1974, p. 624).

En resumen, la elección del emperador en estos momentos de la historia de Roma se lleva a cabo fuera del círculo urbano. A ello colabora de forma directa la provincialización de las legiones. 
El proceso general va desde un emperador senatorial, Galba, pasando por la potencia primordial del pretorio, apoyo de Otón y luego hasta el papel definitivo desempeñado por ejércitos provinciales en el caso de Vitelio. El triunfo posterior de Vespasiano dependerá de su prestigio militar, con lo que consigue el apoyo de distintos grupos del ejército imperial.

Sin entrar en el análisis de los vaivenes militares, sí conviene señalar algo con respecto al Senado (10). Este año de crisis representa el comienzo de una renovación importante no sobre la estructura misma del Senado, sino con respecto al fondo social de sus miembros y del empirismo de la política de transmisión imperial.

\section{c) Soluciones a la crisis}

Si la crisis que estudiamos es fundamentalmente un conflicto de ejércitos, que amenazan constantemente la unidad romana, va a necesitarse, como primer eslabón en la cadena de posibles soluciones, la consecución de la estabilidad total de los militares. En otro orden de cosas se exigirá tenacidad y orden en la nueva planificación socioeconómica.

Ya no es la nobleza de sangre, sino la necesidad de un auténtico orden económico, la que va a prevalecer como el fundamental interés unificador.

En el juego político están en relación, a veces enfrentada, el Senado, los pretorianos y los ejércitos provinciales. Puesto que el Senado está en franca decadencia como consecuencia sobre todo del anterior régimen terrorista, el futuro emperador tendrá que apoyarse necesariamente en un primer momento en los ejércitos provinciales y en los pretorianos.

Restaurar el Imperio, en resumen, y volver a lograr el establecimiento de la auténtica autoridad imperial serán las primeras grandes líneas de la actuación política de Vespasiano, quien inaugura la dinastía de los Flavios. Disciplina y unidad en el ejército y reactivación económica de base presupuestaria e impositiva, serán los puntos concretos de esa política de restauración, que ha de solucionar el período de crisis estudiado.

La salvación estará en una rigurosa y sabia administración. El orden político se restablecerá gracias al acertado programa del nuevo emperador Vespasiano.

\section{BIBLIOGRAFIA}

GARZETTI, A. 1974: From Tiberius to the Antonines. A History of the Roman Empire A. D. 14-192, London, $1 .^{a}$ ed.

HAMMOND, H. 1956: «The transmission of the Power of the Roman Emperor from the Death of Nero in a. D. 68 to that of Alexander Severus in a. D. $235 », M e m$. Am. Ac. Rome, XXIV.

- 1957: «Composition of the Senate A. D. 68-235», JRS XLVII, 74-81.

MANFRE, G. 1947: La crisi politica dell'anno 68-69 d. C. Bologna.

MANNI, E. 1946: «Lotta politica e guerra civile nel 68-69 d. C.», Riv. Fil., XXIV.

MATTINGLY, H. 1926: Coins of the Roman Empire in the British Museum, 1. London.

MC CRUM, M., y WOODHEAD, A. G. 1961: Select Documents of the Principates of the Flavian Emperors, $A$. D. 68-69. Cambridge. 